\title{
Role of delay for the symmetry in the dynamics of networks
}

\author{
O. D'Huys, ${ }^{1}$ I. Fischer, ${ }^{2}$ J. Danckaert, ${ }^{1}$ and R. Vicente ${ }^{3,4}$ \\ ${ }^{1}$ Applied Physics Research Group (APHY), Vrije Universiteit Brussel, 1050 Brussel, Belgium \\ ${ }^{2}$ Instituto de Física Interdisciplinar y Sistemas Complejos, Universidad de las Islas Baleares-Consejo Superior de Investigaciones Cientificas, \\ Campus Universidad de las Islas Baleares, E-07122, Palma de Mallorca, Spain \\ ${ }^{3}$ Max-Planck-Institute for Brain Research, D-60528 Frankfurt am Main, Germany \\ ${ }^{4}$ Frankfurt Institute for Advanced Studies, D-60438 Frankfurt, Germany \\ (Received 8 October 2010; revised manuscript received 21 December 2010; published 26 April 2011)
}

\begin{abstract}
The symmetry in a network of oscillators determines the spatiotemporal patterns of activity that can emerge. We study how a delay in the coupling affects symmetry-breaking and -restoring bifurcations. We are able to draw general conclusions in the limit of long delays. For one class of networks we derive a criterion that predicts that delays have a symmetrizing effect. Moreover, we demonstrate that for any network admitting a steady-state solution, a long delay can solely advance the first bifurcation point as compared to the instantaneous-coupling regime.
\end{abstract}

DOI: 10.1103/PhysRevE.83.046223

PACS number(s): 05.45.Xt, 89.75.Kd, 89.75.Hc, 02.30.Ks

\section{INTRODUCTION}

When an object does not change upon some transformation that object is said to exhibit symmetry. The study of symmetry and related concepts is fundamental in many branches of physics and mathematics. In particular, the symmetry of a dynamic system allows one to predict which type of spatiotemporal patterns can arise in the system (see Ref. [1] and references cited therein). When some parameter is changed transitions between solutions with different degrees of symmetry can occur. Such symmetry-breaking and -restoring bifurcations have received wide attention since they mediate important processes such as the development of biological shapes (morphogenesis) [2,3], the unbalance of left- and right-handed molecules (homochirality) [4], or the secondorder phase transitions underlying critical phenomena such as ferromagnetism and superconductivity [5-7].

Transitions among complex patterns of spatiotemporal dynamics are frequently reported in situations in which similar subsystems interact, thereby forming a discrete network of coupled cells or nodes [8-12]. The physical separation between subsystems can imply some time for the transmission of any effect across them. These propagation times, in addition to other internal temporal latencies, might result in significant interaction delays. Such a coupling delay has a significant effect on the dynamics of, for example, coupled lasers [13], neuronal assemblies, and traffic models [14]. Well-established effects of interaction delays in coupled elements include multistability, the transition to high-dimensional chaos, and oscillation quenching [15-18]. However, there is no clear picture yet about how interaction delays affect the symmetry of the emerging dynamics. Our main motivation is twofold: First we study whether the effects of coupling delays regarding symmetry-breaking bifurcations are topology specific and second we try to obtain a criterion to predict which topologies are sensitive to symmetrizing effects due to the delay and which are not.

Equivariant theory in delayed systems has recently been developed and used in applied mathematics; results include a Lie group classification of second-order delay differential equations [19], an analysis of the bifurcation scenario in a ring of one-dimensional identical units [20], and delayed systems with translational [21] and mirror symmetry [22]. In networks of chaotic delayed-coupling elements it is known that different synchronization patterns appear depending on the coupling topology. For example, a completely synchronized state is unstable for two mutually coupled semiconductor lasers $[23,24]$. When adding a relay, in the form of a semitransparent mirror or a third laser in between the elements [25-27], or when coupling three lasers in a bidirectional ring [28], identical synchrony is stabilized. The same patterns are observed when coupling other systems, such as optoelectronic oscillators [29], chaotic electronic circuits [30], and neuronal assemblies [31]. It has recently been shown that some delayed-coupling chaotic networks can synchronize more easily than others depending only on coupling topology [32].

However, to elucidate if a delay could play different roles for different topologies, it is not enough to compare the synchronous solutions obtained for different delayed-coupling networks. For example, differences across them could stem directly from the restrictions of solutions imposed by the topology and not induced by the delay. Therefore, we focus here on how a given delay modifies the occurrence and location of a bifurcation induced when varying other parameters (in this study the coupling strength between nodes). By comparing the resulting bifurcation scenario between the delayed and the nondelayed cases and doing so for different topologies and different nodes, we clarify how delayed interactions affect the emerging dynamics in relation to the symmetry of the network. Our results include a criterion to predict which topologies are sensitive to symmetrizing effects due to a coupling delay and which are not, independently of the specific dynamics of the coupled elements.

The paper is organized as follows. We start by considering network motifs consisting of linearly coupled Stuart-Landau oscillators. We compare the effects of interaction delays on the dynamics of networks with different symmetry, i.e., unidirectionally and bidirectionally coupled rings. We continue by showing that our results also hold for Mackey-Glass elements, which are linear systems coupled in a nonlinear way. Finally, 
we generalize our results and describe classes of networks for which delay effects are expected to be common within each class.

\section{DELAY EFFECTS IN THE SYMMETRY OF STUART-LANDAU OSCILLATORS}

Let us consider a set of Stuart-Landau oscillators interacting via a linear coupling. The Stuart-Landau oscillator is a generic model for weakly nonlinear oscillators and the normal form of a Hopf bifurcation. When coupled, a network of such oscillators reads

$$
\dot{z_{n}}=z_{n}\left(1-\left|z_{n}\right|^{2}\right)+i \beta z_{n}\left|z_{n}\right|^{2}+\kappa e^{i \theta} \sum_{m} A_{m n} z_{m}(t-\tau),
$$

where the complex variable $z_{n}$ describes the state of the $n$th oscillator and $\beta$ represents a coupling between the amplitude and phase of the oscillator. The coupling between oscillators is described by a strength $\kappa$, a coupling phase $\theta$, and a possible latency $\tau$ representing a propagation delay. The adjacency matrix $A$ accounts for the network topology.

\section{A. Unidirectional rings}

The first network that we analyze is a unidirectional ring of three elements. This motif is invariant under the symmetry group $\mathbb{Z}_{3}$. In this case, the adjacency matrix $A$ is given by

$$
A=\left(\begin{array}{lll}
0 & 1 & 0 \\
0 & 0 & 1 \\
1 & 0 & 0
\end{array}\right) .
$$

To study the role of the delay we start by comparing the dynamics of this network as a function of the coupling strength, for both the instantaneous- and delayed-coupling cases. We notice that for weak coupling both cases (instantaneous and delayed coupling) are well described by their correspondent network of phase oscillators. However, for higher couplings amplitude instabilities develop and retaining the full model, as we analyze it below, is essential to the understanding of the network dynamics. When isolated, each oscillator describes a harmonic motion $z_{j}(t)=r_{0} e^{i \tilde{\omega} t+\phi_{j}}$ with a constant amplitude $r_{0}=1$ and a frequency $\tilde{\omega}=\beta$. Once coupled, the oscillators can adjust their motions and synchronize in phase (i.e., $\phi_{j}=0$ ) with an amplitude and a frequency given, respectively, by

$$
\begin{gathered}
r^{2}=1+\kappa \cos (\theta-\tilde{\omega} \tau), \\
\tilde{\omega}=\beta+\kappa \sqrt{1+\beta^{2}} \sin (\theta+\arctan \beta-\tilde{\omega} \tau) .
\end{gathered}
$$

Besides these in-phase solutions, this network also allows two different antiphase or splay states, where the oscillators exhibit a phase difference of $\pm 2 \pi / 3$ [33]. Here we focus on the symmetric state since its amplitude and frequency are independent of the network structure and therefore it allows for a direct comparison of the effects of delays across different topologies.
For the nondelayed case, the stability of the symmetric (in-phase) state with constant amplitude and frequency can be calculated by a linear stability analysis. The Jacobian to evaluate is given by

$$
J=I_{3} \otimes J_{S}+A \otimes J_{K} .
$$

$J_{S}$ denotes the Jacobian related to the dynamics of each node and $J_{K}$ denotes the Jacobian related to the coupling function. By evaluating Eq. (5) along the eigenvectors of the adjacency matrix $A\left[v_{1}=(1,1,1), v_{2}=\right.$ $\left(1, e^{2 \pi i / 3}, e^{-2 \pi i / 3}\right)$, and $\left.v_{3}=\left(1, e^{-2 \pi i / 3}, e^{2 \pi i / 3}\right)\right]$, it is possible to obtain three simpler equations for the stability along those directions,

$$
J_{n}=J_{S}+\sigma_{n} J_{K}
$$

with $\sigma_{1}=1, \sigma_{2}=e^{2 \pi i / 3}$, and $\sigma_{3}=e^{-2 \pi i / 3}$ the eigenvalues associated with the eigenvectors $v_{1}, v_{2}$, and $v_{3}$, respectively. Thus an instability along the parallel direction $v_{1}=(1,1,1)$ results in a symmetric oscillatory amplitude pattern. In contrast, since the eigenvalues in the two transverse directions are complex conjugates $\left(\sigma_{2}=\sigma_{3}^{*}\right)$, an instability in any of the transverse directions results in a Hopf bifurcation giving rise to rotating waves.

For Stuart-Landau oscillators the characteristic equation [Eq. (6)] is given by

$$
\begin{aligned}
\lambda_{n}= & -r^{2}-\kappa \cos \theta\left(1-\sigma_{n}\right) \\
& \pm \sqrt{r^{4}+4 \beta \kappa \sin \theta\left(1-\sigma_{n}\right)-4 \kappa^{2} \sin ^{2} \theta\left(1-\sigma_{n}\right)^{2}} .
\end{aligned}
$$

Along the synchronization manifold $v_{1}$ there are two possible eigenvalues: $\lambda_{1,1}=0$, which is associated with arbitrary phase shifts, and $\lambda_{1,2}=-2 r^{2}$, whose value is negative independently of any parameter. This implies that the in-phase periodic solution cannot bifurcate into any other symmetric pattern. In transverse directions, however, the system undergoes a supercritical Hopf bifurcation as the coupling strength $\kappa$ increases. Consequently, the amplitude of each oscillator starts fluctuating periodically as shown in the bifurcation diagram in Fig. 1(a). Figure 1(b) display the time traces of simulations of the three oscillators just after this first bifurcation takes place. In that regime both the amplitude and frequency oscillate

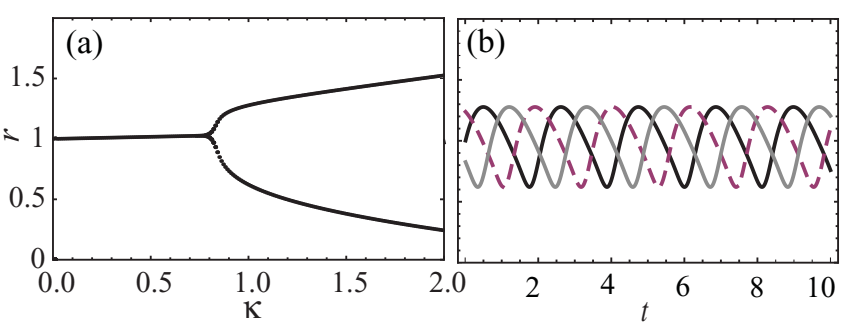

FIG. 1. (a) Bifurcation diagram for three unidirectionally coupled Stuart-Landau oscillators without delay. (b) Amplitude behavior of three unidirectionally coupled Stuart-Landau oscillators after a Hopf bifurcation. The time traces of the different oscillators are plotted as black, gray, and dashed lines. The parameters are $\tau=0, \theta=-1.5$, $\kappa=1$, and $\beta=4$. 
out-of-phase (rotating waves) as predicted by the stability analysis.

The introduction of a coupling delay $\tau$ affects the amplitude and frequency of the in-phase state [Eqs. (3) and (4)] as well as its stability. For the delayed-coupling case the Jacobian now reads

$$
J_{n}=J_{S}+\sigma_{n} e^{-\lambda \tau} J_{K},
$$

which leads to a characteristic equation

$$
\lambda_{n}=-r^{2}-\kappa \cos \Theta\left(1-\sigma_{n} e^{-\lambda \tau}\right) \pm \sqrt{r^{4}+4 \beta \kappa \sin \Theta\left(1-\sigma_{n} e^{-\lambda \tau}\right)-4 \kappa^{2} \sin ^{2} \Theta\left(1-\sigma_{n} e^{-\lambda \tau}\right)^{2}} .
$$

where $\Theta=\theta-\tilde{\omega} \tau$.

Due to the coupling delay, the transverse Hopf bifurcation now occurs for a smaller coupling strength $\kappa$. Such delayinduced advancement of the bifurcating point can be seen in the bifurcation diagram in Fig. 2(a). The incorporation of delay also allows for an instability in the parallel or symmetric direction. However, it can be proved that, for a finite delay, the transverse Hopf instability will always precede the parallel instability [34]. Therefore, for a ring of three unidirectionally coupled oscillators the delay does not alter the amplitude oscillatory pattern observed in the instantaneous case. That is, when the first amplitude instability occurs, independently of the delay, a symmetry-breaking bifurcation results in out-of-phase amplitude oscillations.

We also find that in the presence of coupling delays the first Hopf bifurcation is followed by a second one, eventually leading to quasiperiodic and chaotic dynamics for the amplitude. The oscillatory pattern resulting from the first Hopf bifurcation is maintained across such a cascade: Once the first bifurcation results in out-of-phase amplitude oscillations this signature is carried over to the chaotic regime. This feature is confirmed via a cross-correlation analysis of the chaotic time series for the amplitudes.

\section{B. Bidirectional rings}

Next we study the influence of coupling delays in another network motif: a bidirectional ring of three Stuart-Landau oscillators. This configuration is symmetric under the dihedral group $\mathbb{D}_{3}$. Notice that for three oscillators this network is

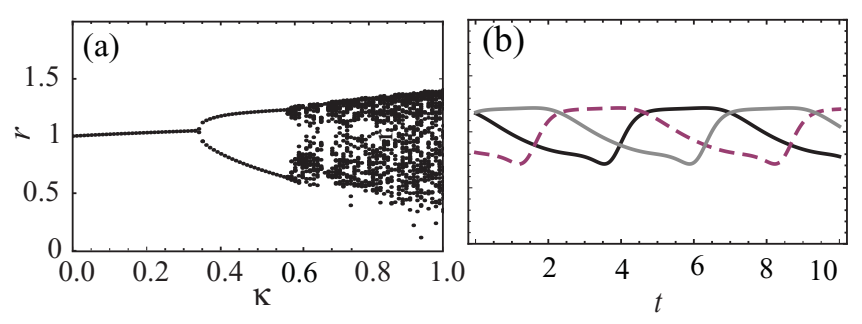

FIG. 2. (a) Bifurcation diagram for three delay-coupled StuartLandau oscillators in a unidirectional ring. (b) Amplitude behavior of three unidirectionally coupled Stuart-Landau oscillators after a Hopf bifurcation. The black, gray, and dashed curves represent the time trace of the three different oscillators. The parameters are $\tau=1.5$, $\theta=-1.5, \kappa=0.5$, and $\beta=4$. equivalent to an all-to-all topology. The adjacency matrix describing the coupling topology is given by

$$
A=\left(\begin{array}{ccc}
0 & \frac{1}{2} & \frac{1}{2} \\
\frac{1}{2} & 0 & \frac{1}{2} \\
\frac{1}{2} & \frac{1}{2} & 0
\end{array}\right) .
$$

The factor $\frac{1}{2}$ compensates for the doubling of incoming connections in comparison with the unidirectional ring. Both networks share the same in-phase periodic state, with the frequency as given in Eq. (4) and the amplitude from Eq. (3), but differ in their symmetry properties. The splay states, which we do not consider, are different from those in a unidirectional ring.

We perform the corresponding stability analysis of the in-phase periodic state for the bidirectional ring. For the instantaneous coupling the Jacobian is still given by Eq. (6). The eigenvectors of the adjacency matrix $\left(v_{1}, v_{2}\right.$, and $\left.v_{3}\right)$ are the same as in a unidirectional ring, however, its eigenvalues are different: $\sigma_{1}=1$ and $\sigma_{2}=\sigma_{3}=-\frac{1}{2}$.

We can immediately see that a bifurcation along the parallel direction is impossible in the bidirectionally instantaneously coupled network (actually, it cannot occur in any network since the eigenvalue along the synchronization manifold is always $\sigma_{1}=1$ ). The eigenvalues of the Jacobian in Eq. (6) are the same in the two transverse directions since $\sigma_{2}=\sigma_{3}$. An ordinary Hopf bifurcation is hence impossible in this network. However, a double Hopf bifurcation, with all four transverse eigenvalues simultaneously crossing the imaginary axis, occurs for increasing coupling strength $\kappa$. This is reflected in the bifurcation diagram in Fig. 3(a). Right after the bifurcation two oscillators synchronize their amplitudes and a third oscillates in antiphase with the two others, with double amplitude. The temporal traces are shown in Fig. 3(b). By increasing the coupling strength further, the three amplitudes each exhibit a different periodic orbit and for even higher coupling, chaotic behavior emerges.

When there is a delay in the coupling, the stability of the periodic state is as described by Eq. (8). By solving the characteristic equation numerically, we find that the first bifurcation point of the in-phase state is again advanced, as in the unidirectional ring. This time, however, for sufficiently long delay, the periodic solution will lose stability first in the in-phase direction via a Hopf bifurcation. Figure 4 depicts the bifurcation diagram and the in-phase oscillations of the amplitude after the Hopf bifurcation. Upon increasing the 


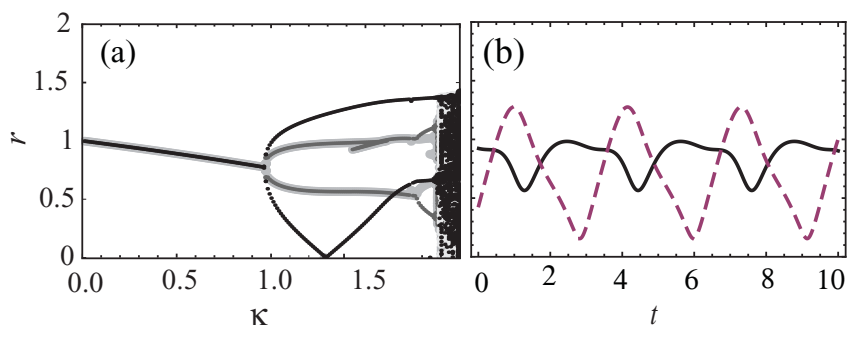

FIG. 3. (a) Bifurcation diagram for three instantaneously coupled Stuart-Landau oscillators in a bidirectional ring. The extrema of the different oscillators are represented in black, light gray, and dark gray. (b) Amplitude behavior of three bidirectionally coupled Stuart-Landau oscillators after a Hopf bifurcation. The black time trace represents a cluster of two oscillators and the dashed time trace represents the amplitude of the third oscillator. The parameters are $\tau=0, \theta=-2, \kappa=1.4$, and $\beta=4$.

coupling strength further, the oscillators remain synchronized, even in the quasiperiodic and chaotic regime. (Short parameter ranges may exist where the symmetry breaks slightly, but in the chaotic regime it is always restored. We note that out-of-phase oscillations are also possible when bifurcating from the splay states.)

Thus we have seen that a coupling delay induces common effects in both ring configurations, in particular the advancing of the first bifurcating point. In addition, we have found effects that are specific to the network. In particular, the incorporation of delay in the bidirectional ring alters the symmetry of the pattern of oscillations compared to its instantaneous counterpart. This is not the case for the unidirectional ring, where the oscillatory pattern remains identical regardless of whether or not a coupling delay is taken into consideration. We also compared four Stuart-Landau oscillators, coupled in a unidirectional ring and in an all-to-all configuration, and we found similar results: In both coupling topologies the delay advances the bifurcation point, but only when the four oscillators are globally coupled does the delay have an effect on the oscillation pattern.

\section{DELAY EFFECTS IN THE SYMMETRY OF MACKEY-GLASS OSCILLATORS}

To further investigate whether the relation between symmetry and delay effects depends on the internal dynamics of the nodes we replace the Stuart-Landau oscillators with
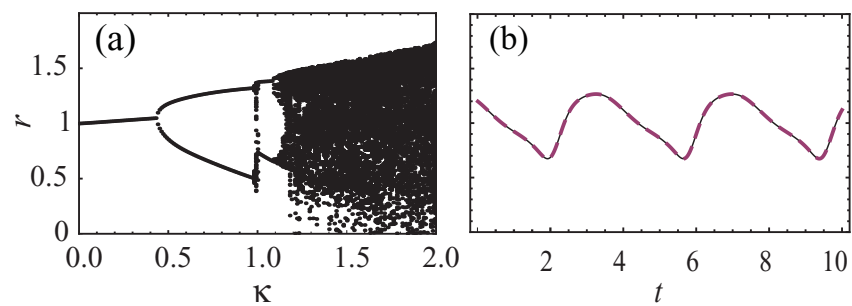

FIG. 4. (a) Bifurcation diagram for three delayed-coupling Stuart-Landau oscillators in a unidirectional ring. (b) Amplitude behavior of three unidirectionally coupled Stuart-Landau oscillators after a Hopf bifurcation. The parameters are $\tau=3, \theta=-2, \kappa=0.7$, and $\beta=4$.
Mackey-Glass elements. This type of oscillator was introduced by Mackey and Glass to study physiological control systems [35], such as the production of blood cells. We consider the following model equation a network of Mackey-Glass oscillators [35]:

$$
\dot{x}_{n}=-x_{n}+a \frac{\sum A_{m n} x_{m}(t-\tau)}{1+\left[\sum A_{m n} x_{m}(t-\tau)\right]^{c}},
$$

where $a>0$ is the connection strength and $c$ is the degree of the nonlinearity. While in a network of Stuart-Landau oscillators the nonlinear nodes are linearly coupled, we consider here a network of linear nodes coupled in a nonlinear manner.

\section{A. Unidirectional rings}

We first study a unidirectional ring of three instantaneously coupled Mackey-Glass elements. Since the nodes are described by a scalar variable, it is possible to treat the system fully analytically. The bifurcation diagram is shown in Fig. 5(a). For small coupling $a<1$, the zero state is stable, until at $a=1$ it becomes unstable (in a transcritical bifurcation) in the parallel direction and the elements reach a symmetric nonzero steady state

$$
x_{1}=x_{2}=x_{3}=(a-1)^{1 / c} .
$$

The next instability occurs in the transverse directions; at

$$
a=\frac{c \cos 2 \pi / 3}{1+\cos 2 \pi / 3(c-1)}
$$

the system undergoes a Hopf bifurcation, leading to out-ofphase oscillations, as shown in Fig. 5.

Adding a delay in the coupling, this Hopf bifurcation in the transverse direction advances toward

$$
a=\frac{c}{c-2} \text {. }
$$

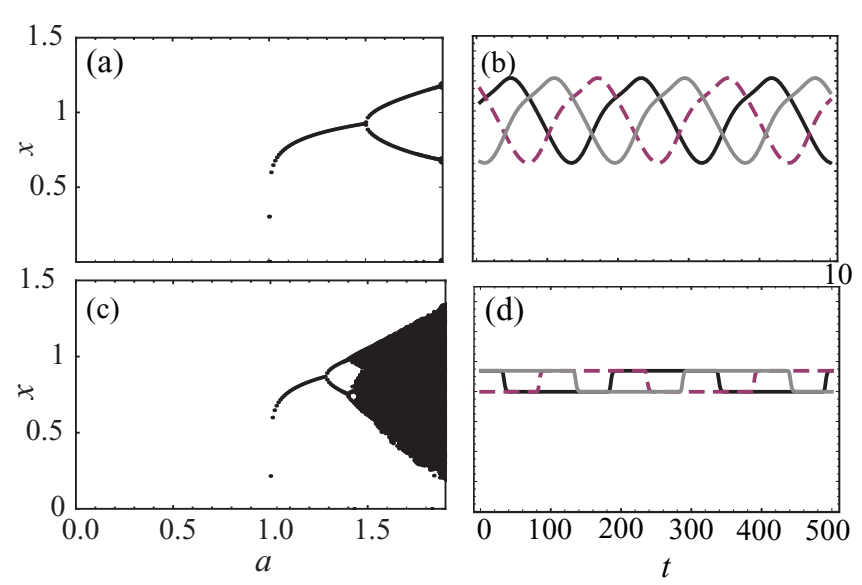

FIG. 5. (a) and (c) Bifurcation diagrams for varying coupling $a$ and (b) and (d) examples of a time trace for three unidirectionally coupled Mackey-Glass elements (a) and (b) without and (c) and (d) with delay. The time traces of the three elements are plotted as black, gray, and dashed lines. The parameters are (a) and (b) $c=9$ and $\tau=0$, (c) and (d) $\tau=50$, and (b) and (d) $a=1.33$. 


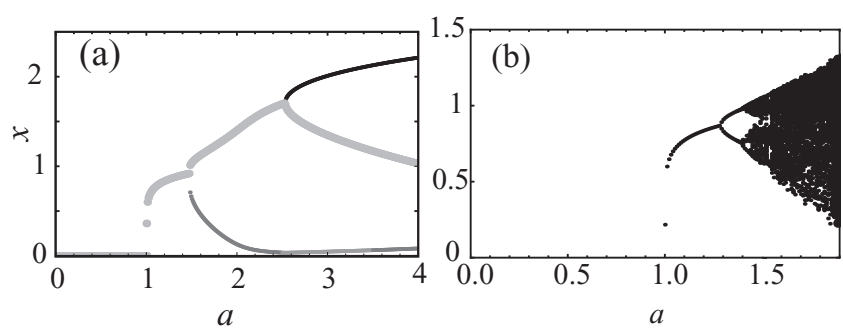

FIG. 6. Bifurcation diagram for (a) three mutually instantaneously coupled and (b) delayed-coupling Mackey-Glass elements. The extrema of the different elements are plotted in black, dark gray, and light gray.

The bifurcation diagram is shown in Fig. 5(c). Just like in a network of Stuart-Landau oscillators, a Hopf bifurcation in the parallel direction is possible due to the delay, but the transverse Hopf bifurcation always precedes the parallel one. Consequently, also in a network of unidirectionally coupled Mackey-Glass elements the introduction of a coupling delay does not affect the oscillation pattern.

\section{B. Bidirectional rings}

If the three elements are connected bidirectionally, the coupling delay does have an effect on the synchronization behavior of the network. Without delay, the network undergoes a (subciticritical) pitchfork bifurcation at

$$
a=\frac{c \cos 2 \pi / 3}{1+(c-1) \cos 2 \pi / 3}
$$

in transverse directions, resulting in a cluster of two elements, while the third one has a different constant value. A Hopf bifurcation cannot occur without coupling delay if all connections in a network are bidirectional since the nodes are one-dimensional systems; the Jacobian then becomes a real and symmetric matrix. With the introduction of a latency, the system undergoes a Hopf bifurcation at

$$
a=\frac{c}{c-2},
$$

resulting in in-phase oscillations. The bifurcation diagrams of three oscillators coupled bidirectionally with and without delay are shown in Fig. 6.

\section{GENERAL RESULTS FOR ARBITRARY TOPOLOGIES AND OSCILLATORS}

In the following we discuss to what extent these findings can be generalized. First we demonstrate the destabilizing effect in the long-delay limit. We start from an arbitrary system, which can be a network, with delay, allowing for a steady state. By performing a linear stability analysis, we can write the characteristic equation for this equilibrium as

$$
F\left(\lambda, e^{-\lambda \tau}\right)=0 \text {. }
$$

Without delay, we assume that this steady state loses stability as a function of a system parameter. At the bifurcation point we can write

$$
F\left(i \omega_{0}, 1\right)=0 .
$$

The solutions of equations of the type of Eq. (12) have been analyzed in the long-delay limit by Yanchuk et al. (see
Ref. [36] and references cited therein). They find that some of the eigenvalues, the so-called strongly unstable spectrum, have a positive real part that does not scale with the delay. For steady states, which are stable without delay, such strongly unstable eigenvalues do not exist. The other eigenvalues, which are called the pseudocontinuous spectrum, can be estimated as

$$
\lambda=i \omega+\frac{\gamma}{\tau}+O\left(\frac{1}{\tau^{2}}\right) .
$$

By defining

$$
Y(\omega)=e^{-\lambda \tau}
$$

one can write the characteristic equation as $F(i \omega+\gamma / \tau, Y)=0$. By neglecting the terms inversely proportional to the coupling delay, it is possible to solve the characteristic equation graphically and to obtain a curve $\gamma(\omega)=-\ln |Y(\omega)|$, on which the eigenvalues lie densely in the long-delay limit.

By evaluating this curve at $\omega_{0}$, the Hopf frequency without delay, we find that $Y\left(\omega_{0}\right)=1$ and hence $\gamma\left(\omega_{0}\right)=0$. The solution of the characteristic equation at the bifurcation points without delay is part of the pseudocontinuous spectrum and is thus also infinitely close to the eigenvalues in the long-delay limit. This implies that generically, as a function of a system parameter, some eigenvalue for the long-delay case will cross the imaginary axis before the eigenvalue associated with the zero-delay coupling. Consequently, a long delay can only advance a bifurcation point independent of the system.

To study the influence of network topology, we assume a network of identical delayed-coupling elements, where the type of connections is the same in the network. The strength of the connections may differ, but we assume that each element receives the same amount of input such that the network can synchronize identically. We consider a symmetric steadystate solution of such a network, which changes stability as a function of a parameter. Under these conditions, it is possible to split the characteristic equation along the eigenvectors $v_{k}$ of the adjacency matrix. We can write these equations as

$$
F\left(\lambda_{k}, \sigma_{k} e^{-\lambda \tau}\right)=0 .
$$

As before, $\sigma_{k}$ are the eigenvalues of the adjacency matrix.

In any network, along the synchronization manifold, the eigenvalue of the adjacency matrix is $\sigma_{1}=1$. By defining $Y=\sigma_{1} e^{-\lambda \tau}=e^{-\lambda \tau}$, the parallel pseudocontinuous spectrum is then given by $\gamma_{1}(\omega)=-\ln |Y(\omega)|$. In transverse directions, we follow the same procedure, but this time with $Y=\sigma_{k} e^{-\lambda \tau}$.

For Stuart-Landau oscillators the characteristic equation then reads

$$
\begin{aligned}
0= & i \omega-r^{2}-\kappa \cos \Theta(1-Y) \\
& \pm \sqrt{r^{4}+4 \beta \kappa \sin \Theta(1-Y)-4 \kappa^{2} \sin ^{2} \Theta(1-Y)^{2}}
\end{aligned}
$$

in both the parallel and transverse directions. The difference in stability between the parallel and transverse directions depends on the relation of $Y$ and $\gamma$.

We find that

$$
\begin{aligned}
\gamma_{k}(\omega) & =-\ln |Y(\omega)|+\ln \left|\sigma_{k}\right| \\
& =\gamma_{1}(\omega)+\ln \left|\sigma_{k}\right| .
\end{aligned}
$$




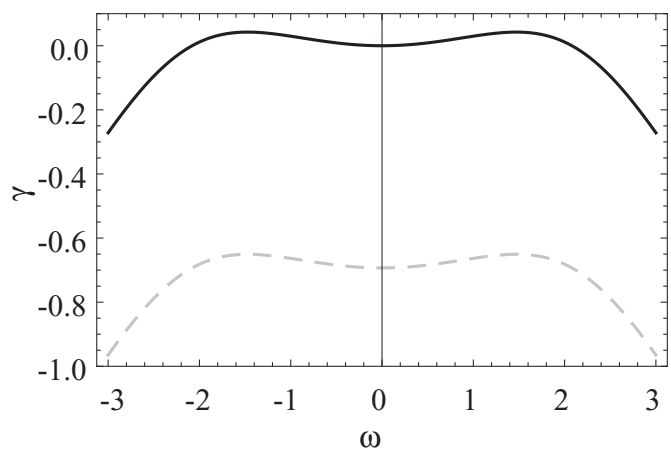

FIG. 7. Parallel (solid line) and transverse (dashed line) pseudocontinuous spectra of three bidirectionally coupled Stuart-Landau oscillators after a Hopf bifurcation. The parameters are $\Theta=-2 \pi / 5$, $\kappa=1$, and $\beta=3$.

Since in all networks $\left|\sigma_{k}\right| \leqslant 1$ holds, we find that some transverse spectra $\gamma_{k}(\omega)$ are downshifted with respect to the parallel spectrum $\gamma_{1}(\omega)$ and some are equal to the parallel spectrum. An example is shown in Fig. 7. Consequently, the first bifurcation point (i.e., the set of parameters for which the parallel pseudocontinuous spectrum touches the imaginary axis) is the same in all networks.

We note that for a unidirectional ring, the bifurcations in the long-delay limit occur for the same parameters as in the case where infinitely many elements are coupled without delay. This result was shown by Perlikowski et al. [37] in the specific case of Stuart-Landau oscillators; we show here that it is valid for a broad class of oscillators. For a bifurcation (in the parallel direction) in a system with a delay $\tau^{*}$, with Hopf frequency $\omega^{*}$, we have $F\left(i \omega^{*}, e^{i \psi}\right)=0$ with $\psi=-\omega^{*} \tau^{*}$. Since in a unidirectional ring the eigenvalues of the adjacency matrix are given by $\sigma_{k}=e^{2 k \pi i / N}$, with $N$ the number of elements, $e^{i \psi}$ is an eigenvalue and the bifurcation will occur for the same parameters. Consequently, due to the delay, the bifurcation point in any network shifts toward the bifurcation point in an infinite unidirectional ring.

Thus Eq. (16) allows us to distinguish between networks where the delay has a symmetrizing effect and where it does not. If any eigenvalue lies on the unit cycle $\left|\sigma_{k}\right|=1$, as is the case, e.g., in unidirectional rings or for bipartite networks, we have $\gamma_{k}(\omega)=\gamma_{1}(\omega)$. In these networks the delay does not necessarily affect the synchronization properties of the network. In contrast, if for all eigenvalues $\left|\sigma_{k}\right|<1$ holds, all transverse pseudocontinuous spectra are downshifted with respect to the spectrum in the parallel direction and, consequently, all bifurcations will occur along the synchronization manifold. Examples of such graphs are globally coupled networks and bidirectional rings with an odd number of elements.
If not all network nodes receive the same amount of input (i.e., there is a mismatch of coupling strengths), an identical synchronized state is not a solution of the system. However, we can often still follow the same approach and identify eigenvectors and eigenvalues of the (total) Jacobian; hence we can predict the (approximate) pattern after the bifurcation. If the mismatch is small, the eigenvalues and eigenvectors are close to their unmismatched counterparts and similar conclusions hold. A mismatch in the coupling delays can have a more significant influence on the oscillation pattern. For example, with appropriately chosen delays, one can obtain patterns where one node is lagging another with a time difference equal to the delay difference [29]. Depending on the ratio between the different delays, different synchronization patterns can be observed in a network of chaotic elements [38]. Also the dynamics of a system with multiple delays can differ from systems with a single one: It has been shown, for example, that a laser subject to two different delays may stabilize its dynamics $[39,40]$. In contrast, in a unidirectional ring only the total round-trip delay and the delays between the different elements play a role in the dynamics (and symmetry) [41]. Overall, the effect of delay mismatches depends strongly on the number of mismatches and the network topology, but we expect the strongest effect on frequencies around $n / 2 \Delta \tau$ in the pseudocontinuous spectrum (where $\Delta \tau$ refers to the delay difference). We do not expect that noise has a major influence on the role of the delay. We defer to further investigation a more specific analysis of these problems.

\section{CONCLUSION}

In conclusion, we have demonstrated that delay has some universal effects in the sense that they are common to any system admitting a symmetric steady state. Specifically, we found the advancing of the bifurcating point to the coupling delay. Additional effects, such as the symmetrizing role of delays on network dynamics, is specific to some topologies. This shows that the ways in which a delay modifies the spatiotemporal patterns of a network is interrelated with the very same network and cannot be decoupled.

\section{ACKNOWLEDGMENTS}

O.D. acknowledges the Research Foundation Flanders (FWO-Vlaanderen) for support. This work was partially supported by the Interuniversity Attraction Poles program Photonics@be of the Belgian Science Policy Office under Grant No. IAP VI-10 by MICINN (Spain) under Project No. DeCoDicA (TEC2009-14101) and by the project PHOCUS (EU FET-Open Grant No. 240763). S. Yanchuk and P. Perlikowski are gratefully acknowledged for fruitful discussions.
[1] M. Golubitsky and I. Stewart, The Symmetry Perspective (Birkhäuser, Basel, 2003).

[2] S. Kondo and R. Asai, Nature (London) 376, 765 (1995).

[3] A. M. Turing, Philos. Trans. R. Soc. London Ser. B 237, 37 (1952).
[4] D. K. Kondepudi, R. J. Kaufman, and N. Singh, Science 250, 975 (1990).

[5] L. D. Landau and E. M. Lifshitz, Statistical Physics, 3rd ed., Part 1, Vol. 5 of Course of Theoretical Physics (Pergamon, New York, 1994). 
[6] R. D. Averitt, Nature Phys. 6, 639 (2010).

[7] R. Yusupov, T. Mertelj, V. V. Kabanov, S. Brazovskii, P. Kusar, J.-H. Chu, I. R. Fisher, and D. Mihailovic, Nature Phys. 6, 681 (2010).

[8] J. J. Collins and I. N. Stewart, J. Nonlinear Sci. 3, 349 (1993).

[9] K. Ito and Y. Nishiura, Phys. Rev. E 77, 036224 (2008).

[10] J. Kestler, W. Kinzel, and I. Kanter, Phys. Rev. E 76, 035202 (2007).

[11] S. A. Campbell, Y. Yuan, and S. D. Bungay, Nonlinearity 18, 2827 (2005).

[12] L. Chen and K. Aihara, IEEE Trans. Circuits Syst. I 49, 602 (2002).

[13] R. Lang and K. Kobayashi, IEEE J. Quantum Electron. 16, 347 (1980).

[14] G. Orosz, R. E. Wilson, R. Szalai, and G. Stépan, Phys. Rev. E 80, 046205 (2009).

[15] M. Dhamala, V. K. Jirsa, and M. Ding, Phys. Rev. Lett. 92, 074104 (2004).

[16] D. V. Ramana Reddy, A. Sen, and G. L. Johnston, Phys. Rev. Lett. 85, 3381 (2000).

[17] T. Erneux, Applied Delay Differential Equations (Springer, New York, 2009).

[18] S. Yanchuk and P. Perlikowski, Phys. Rev. E 79, 046221 (2009).

[19] P. Pue-on, Ph.D. thesis, Suranaree University of Technology, 2007.

[20] D. Fan and J. Wei, Math. Prob. Eng. 2009, 950251 (2009).

[21] G. Orosz and G. Stépán, J. Nonlinear Sci. 14, 505 (2004).

[22] B. F. Redmond, V. G. LeBlanc, and A. Longtin, Physica D 166, 131 (2002).

[23] T. Heil, I. Fischer, W. Elsäßer, J. Mulet, and C. R. Mirasso, Phys. Rev. Lett. 86, 795 (2001).

[24] S. Tang, R. Vicente, M. Chiang, C. Mirasso, and J. Liu, IEEE J. Sel. Top. Quantum Electron. 10, 936 (2004).
[25] I. Fischer, R. Vicente, J. M. Buldu, M. Peil, C. R. Mirasso, M. C. Torrent, and J. Garcia-Ojalvo, Phys. Rev. Lett. 97, 123902 (2006).

[26] E. Klein, N. Gross, M. Rosenbluh, W. Kinzel, L. Khaykovich, and I. Kanter, Phys. Rev. E 73, 066214 (2006).

[27] A. S. Landsman and I. B. Schwartz, Phys. Rev. E 75, 026201 (2007).

[28] J. Buldu, M. Torrent, and J. Garcia-Ojalvo, J. Lightwave Technol. 25, 1549 (2007).

[29] M. Peil, L. Larger, and I. Fischer, Phys. Rev. E 76, 045201 (2007).

[30] A. Wagemakers, J. Buldu, and M. Sanjuan, Chaos 17, 023128 (2007).

[31] R. Vicente, L. Gollo, C. Mirasso, I. Fischer, and G. Pipa, Proc. Natl. Acad. Sci. USA 105, 17157 (2008).

[32] V. Flunkert, S. Yanchuk, T. Dahms, and E. Schöll, Phys. Rev. Lett. 105, 254101 (2010).

[33] C. U. Choe, T. Dahms, P. Hövel, and E. Schöll, Phys. Rev. E 81, 025205(R) (2010).

[34] O. D'Huys, R. Vicente, J. Dankaert, and I. Fischer, Chaos 20, 043127 (2010).

[35] M. C. Mackey and L. Glass, Science 197, 287 (1977).

[36] S. Yanchuk, M. Wolfrum, P. Hövel, and E. Schöll, Phys. Rev. E 74, 026201 (2006)

[37] P. Perlikowski, S. Yanchuk, O. V. Popovych, and P. A. Tass, Phys. Rev. E 82, 036208 (2010).

[38] A. Englert, W. Kinzel, Y. Aviad, M. Butkovski, I. Reidler, M. Zigzag, I. Kanter, and M. Rosenbluh, Phys. Rev. Lett. 104, 114102 (2010).

[39] F. Rogister, P. Mégret, O. Deparis, M. Blondel, and T. Erneux, Opt. Lett. 24, 1218 (1999).

[40] Y. Liu and J. Ohtsubo, IEEE J. Quantum Electron. 33, 1163 (1997).

[41] G. Van der Sande, M. C. Soriano, I. Fischer, and C. R. Mirasso, Phys. Rev. E 77, 055202 (2008). 